\title{
Gender balancing staff recruitment: attracting the right candidates
}

Lara Isbel \& Judy Robertson

The University of Edinburgh established the Chancellor's Fellowship scheme in 2012 to recruit 100 people with the potential to be future research leaders in their fields. Two-thirds of the first intake of Chancellor's Fellows were men. However, two-thirds of the people who applied to the scheme were also men: it is very challenging to address gender imbalances internally if the starting point is a very unbalanced pool. The same is true for recruitment to our Information Services division where the University faces many of the same challenges as other tech employers in Edinburgh.

In fields where there is a significant gender imbalance, such as computer science or nursing, getting a more diverse pool of qualified people to apply isn't easy.

This recipe provides some practical steps which may help you to promote job vacancies to reach a wider pool of people with relevant experience and qualifications.

\section{Ingredients}

- Critical perspective.

- Openness to ideas.

- An awareness of your own bias.

- Willingness to experiment with and evaluate different approaches.

\section{Method}

1. Write an inclusive job advert. Job seekers interpret subtle linguistic clues to decide whether they would "belong" within a particular working environment. Women are less likely to find it appealing to apply for jobs based on adverts which are full of words with masculine associations, regardless of whether they think they could do the job. If you're wondering what masculine words are, consult the appendix of Gaucher et al. (2011) for a handy list from the literature. As a small sample, "decisive", "ambitious", "intellectual" and "adventurous" are all words more associated with men. Yes, men get the good words, and they get Lego. There really is no justice.

So-called feminine words include "loyal", "co-operative" and "responsible" and a whole lot of other words which might be better associated with dogs like "affectionate" and "submissive". Also be aware that within academic fields (such as philosophy, maths and physics) where women are under-represented, success is associated with innate talent rather than hard work, (Leslie et al., 2015). Advertising for a "brilliant" professor is unlikely to improve gender balance in these domains.

2. Pay attention to how the salary is described. Women are more inclined to negotiate salary if the advert gives them "permission" to do so by stating "salary 


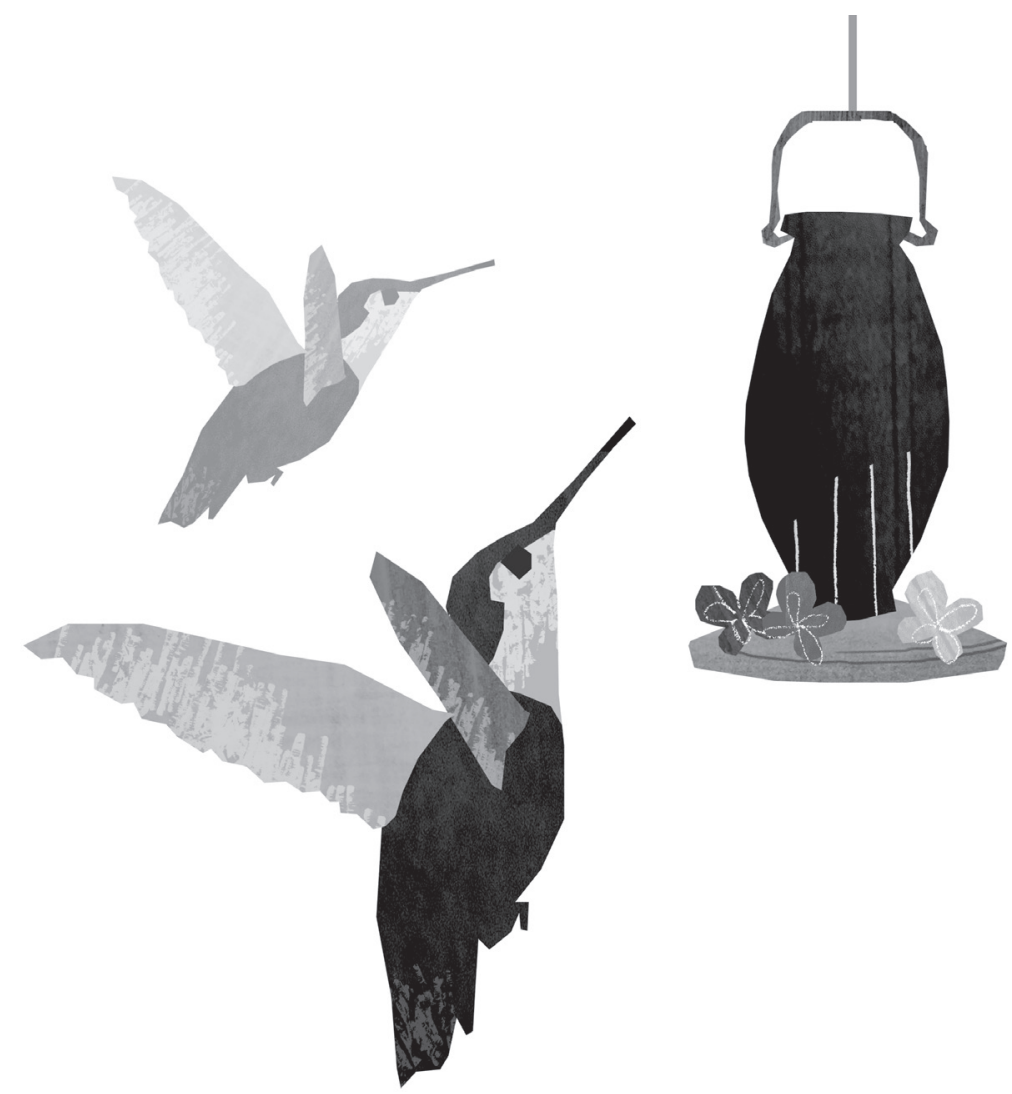

negotiable" or including a range on a salary scale (see Salary negotiation). This detail is important to avoid perpetuating the gender pay gap. Adverts which frame a salary in competitive terms are off-putting to both men and women, but will deter women more.

For example, if the salary scheme links the applicant's pay to performance in comparison to colleagues, women will be less likely to apply, although this effect can be mitigated by making the language in the advert more gender neutral (Gaucher et al., 2011).

3. Include in the job information links to the University's family-friendly policies, flexible working opportunities, facilities, support for LGBTO+ (lesbian, gay, bisexual, trans, queer/questioning and other) staff and other information which might make the job more attractive to a wider range of candidates. To attract a diversity of talent, the University needs to make itself attractive to candidates who have a life outside academia. These include people with family responsibilities such as caring for young children or ageing parents.

4. Have visible role models. For a large recruitment campaign, you could include short profiles or miniinterviews with people from a broad range of backgrounds who are in similar roles to the post advertised.

5. Ask for advice from HR (Human Resources) on where to advertise the vacancy and ask candidates how they heard about the job to check which channels are most effective. Experiment with different routes and social media to see if that has an impact on the balance of applications. 
6. Ask current staff members to share the vacancy with their networks and to encourage talented contacts (of both genders) to apply. If someone feels like a role is 'not for them', encouragement or support from a third party who is aware of their professional expertise may increase the likelihood of them applying.

\section{Check that the evidence you ask for} matches the qualities and experience you require. Make sure that the essential criteria are well thought through (see Defining excellence).
8. Check that your recruitment process once applications are received follows best practice. This involves shortlisting (see Gender balancing staff recruitment: shortlisting) and selection (see Gender balancing staff recruitment: interviewing). Consider which aspects of the role you can check at the application stage and what to look for in an interview. Structured interview questions will help you decide whether each candidate meets your criteria. For example, if the ability to perform the duties of a personal tutor is required, ask for examples of prior experience at interview because it is unlikely to be included in the standard academic CV.

In the Schools of Molecular, Genetic and Population Health Sciences, and Clinical Sciences, all job adverts include a statement which welcomes applications from women. In the School of Health in Social Science at Edinburgh University, it is routine to advertise that posts can be part-time or flexible. 